\title{
Radiologic and Pathological Investigation of Pseudarthrosis in Ankylosing Spondylitis: Distinguishing Between Inflammatory and Traumatic Etiology
}

\author{
Mu Qiao, Bang-ping Qian, Yong Qiu, Sai-Hu Mao, and Yi-hua Wang
}

\begin{abstract}
Objective. To investigate the pathogenesis of pseudarthrosis in ankylosing spondylitis (AS) based on the pathological analysis of specimens harvested during surgery.

Methods. Radiographic and clinical data for 17 consecutive AS patients with pseudarthrosis were retrospectively analyzed. Meanwhile, the pathological analysis of specimens obtained during surgery was also performed.

Results. In total, 18 extensive Andersson lesions were included. Pseudarthrosis located at the apical region were noted in 12 patients. Complete ossified anterior longitudinal ligaments above or below pseudarthrosis and fracture through posterior elements or facet joints were observed in 7 and 6 lesions, respectively. The most definitive pathological characteristic in all cases was proliferating hypovascular edematous fibrous tissue involving disc, bone-disc border, and vertebral body. Fibrinoid necrosis, necrotic bone fragments, hemosiderin deposits, and active subchondral osteogenesis were found, indicating trauma process. Mild perivascular collections of inflammatory cells were detected in only 2 cases.

Conclusion.AS-related pseudarthrosis is more likely to originate from mechanical trauma than inflammation. The above-mentioned radiological and histological findings showed that multiple mechanisms lead to the formation of pseudarthrosis. These mechanisms include excessive stress, insufficiency fracture, and an acute fracture involving a 3-column structure. (First Release September 15 2018; J Rheumatol 2019;46:259-65; doi:10.3899/jrheum.171249)
\end{abstract}

Key Indexing Terms:

PSEUDARTHROSIS PATHOLOGICAL SECTION
ANKYLOSING SPONDYLITIS
PATHOGENESIS TRAUMATIC
Ankylosing spondylitis (AS) is a common chronic inflammatory disorder that mainly affects the spinal column and sacroiliac joints ${ }^{1}$. In the advanced stage of AS, progressive ossification often produces a fixed and rigid thoracolumbar kyphotic deformity. Spinal pseudarthrosis, a well-known complication of AS, was first described by Andersson in 1937

From Spine Surgery, and the Department of Pathology, Nanjing Drum Tower Hospital, the Affiliated Hospital of Nanjing University Medical School; Medical School of Southeast University, Nanjing, China.

Financial support from the Key Provincial Talents Program of Jiangsu Province, the Jiangsu Provincial Key Medical Center (ZX201107), and the National Natural Science Foundation of China (81372009).

M. Qiao, MD, Spine Surgery, Nanjing Drum Tower Hospital, the Affiliated Hospital of Nanjing University Medical School, and Medical School of Southeast University; B.P. Qian, MD, Spine Surgery, Drum Tower Hospital of Nanjing University Medical School; Y. Qiu, MD, Spine Surgery, Drum Tower Hospital of Nanjing University Medical School; S.H. Mao, MD, Spine Surgery, Drum Tower Hospital of Nanjing University Medical School; Y.H. Wang, MD, Department of Pathology, Drum Tower Hospital of Nanjing University Medical School.

Address correspondence to Dr. B.P. Qian, Spine Surgery, Drum Tower Hospital of Nanjing University Medical School, Zhongshan Road 321, Nanjing 210008,China.E-mail: qianbangping@163.com

Accepted for publication June 21, 2018. (named Andersson lesion; AL) ${ }^{2}$. In 1996, an epidemiological investigation of 147 consecutive AS patients was performed by Kabasakal, et $a l^{3}$. In that study, 12 individuals (8\%) with spondylodiscitis involving 32 disc spaces were identified. In 2000, Scarpa $^{4}$ evaluated the destructive abnormalities in psoriatic arthritis following Cawley's classifications. Thus, destructive erosions and lesions of the discovertebral junction were identified in 12 patients (12.0\%) and seemed to be a characteristic aspect of spondyloarthropathies. Since then, the different terms that have been used to refer to these lesions, including spondylodiscitis, destructive lesion, discitis, or pseudarthrosis, reflect the existence of an ongoing debate regarding the exact etiology of $\mathrm{AL}^{2,5,6,7}$.

Before the 1970s, the term spondylodiscitis was used to describe an inflammation-related etiology of AL. Wholey, et $a l^{5}$ was the first to describe findings in biopsies obtained from 2 patients with AS, stating that the observed changes were consistent between the samples and indicated a chronic inflammatory process. In a more detailed pathological investigation of 1 patient with AS, Hansen, et $a l^{6}$ reported the finding of nonspecific granulomatous inflammatory tissue, also favoring a purely inflammatory origin. However, the

Personal non-commercial use only. The Journal of Rheumatology Copyright $\odot$ 2019. All rights reserved. 
relevant changes documented by other authors have included the replacement of bone by fibrous tissue or hemosiderin deposits, both of which indicate that traumatic factors might be involved in the pathogenesis of $\mathrm{AL}^{7}$. Thus, a theory that combined inflammatory processes with mechanical effects on the spinal column became more widely accepted in the early 1980s. Nevertheless, in a study of 35 patients with AS published by Fang, et al, trauma without an inflammatory element was assumed to be the primary cause of extensive lesions based on detailed histological observations of 8 specimens ${ }^{8}$. Because the above-mentioned studies of pseudarthrosis complicated with AS are largely limited to isolated case reports, a more detailed and comprehensive analysis of pathogenesis is clearly needed. The purpose of our study was to investigate histological findings in radiologic examinations and determine the exact etiology underlying pseudarthrosis.

\section{MATERIALS AND METHODS}

Medical records of 75 consecutive AS patients with pseudarthrosis who underwent surgery between January 2001 and December 2016 were reviewed. The inclusion criteria were the following: (1) radiograph, computed tomography (CT), and magnetic resonance imaging (MRI) data were available; and (2) tissue specimens around pseudarthrosis were obtained intraoperatively. Tissue specimens could be obtained in patients by osteotomy through pseudarthrosis, debridement, or decompression around the pseudarthrosis, instead of posterior instrumentation only. Finally, 17 patients with 18 extensive vertebral lesions were included in our study. In this paper, regarding the location of pseudarthrosis, apex was defined as "apical region of global kyphosis (GK)." Meanwhile, above and below were defined as "above the apex of GK" and "below the apex of GK," respectively. The other 58 patients were excluded because the detected pseudarthroses were not located at the apical region of GK, and severe spinal cord impingement was not identified in these cases; thus, debridement or decompression around the lesions were not performed and tissue specimens were not available (Supplementary Table 1, available with the online version of this article). All lesions were confirmed on anteroposterior and lateral radiographs, CT, and MRI. An excision of the intervertebral disc and part of the adjacent vertebral body was achieved intraoperatively. GK was defined as the "angle between the superior endplate of the maximally tilted upper end vertebra and the inferior endplate of the maximally tilted lower end vertebra on full-length lateral radiographs. ${ }^{1}$ " GK $>50^{\circ}$ was regarded as indicative of thoracolumbar kyphosis. This study was approved by the Medical Ethics Committee of our hospital (ethics approval number 2011052), and written informed consent was obtained from all patients prior to testing.

Clinical features. At the time that pseudarthrosis was documented, 12 patients showed rigid thoracolumbar kyphosis. Persistent localized back pain around the lesion was noticed and these symptoms were aggravated when walking but relieved after lying down. The neurological functioning of 17 patients is listed in Table 1. It should be noted that pseudarthrosis frequently involves 3 columns of the spine and is at high risk of spinal instability, thereby leading to the compression of the spinal cord. Thus, surgical intervention should be taken into consideration immediately if patients have progressive myelopathy symptoms and neurological deficit secondary to the spinal instability of those pseudarthrotic lesions. All 17 patients included received only nonsteroidal antiinflammatory drugs (NSAID) to reduce the abnormal inflammatory markers to normal values before surgery. In our present study, the administration of NSAID preoperatively is aimed to relieve the pain and provide a favorable condition for surgery. Recently, Molnar, et $a l^{9}$ analyzed 432 patients with AS for the effect of tumor necrosis factor inhibitors on spinal structural changes. They concluded that the structural damage and radiographic progression can be suppressed only if the treatment with antiinflammatory drugs were used for over 2 years. Also, Haroon, et $a l^{10}$ demonstrated that high NSAID intake for more than 2 years may show benefits for the progression of structural changes in patients with AS. To our knowledge, pseudarthrosis is known as a late complication for AS patients with a longstanding disease course. Thus, the application of antiinflammatory medication for a short period before surgery may not have an effect on the biopsy. No evidence of bacterial infection or tuberculous spondylitis was detected in these patients.

Treatment. Four surgical strategies were adopted for these 17 patients (Table 1).

\section{RESULTS}

A total of 14 men and 3 women ranging in age from 29 to 60 years were included. Radiographs and CT scans were available in all cases, while MRI data were obtained for 13 of the patients (76\%). For the 2 surgical specimens from the same patient, the radiological and pathological manifestations of the lesion were identical.

Radiographs. The average preoperative GK was $69.4^{\circ} \pm$ $24.8^{\circ}$ (range $32^{\circ}$ to $114^{\circ}$ ). Twelve patients were regarded as having thoracolumbar kyphosis. All lesions were observed in the thoracolumbar and lumbar region between T9 and L5. Lesions located at the apex of thoracolumbar kyphosis were noted in 12 patients. The most striking radiographic feature of pseudarthrosis is osteolytic destruction of the vertebral endplate and adjoining reactive sclerosis extending into the vertebral bodies. In 7 lesions, bridging syndesmophytes were noted above and below the pseudarthrosis. A fracture through the posterior elements or unfused facet joints combined with opposite anterior lesions were observed in 6 patients.

CT scans. Irregular central discovertebral osteolysis with a reactive sclerotic zone was identified in nearly all the lesions. Vacuum phenomenon and paraspinal swellings were noted in 11 lesions. Lesions extending into the posterior elements led into the narrow of the spinal canal in 3 cases. Among these lesions, vertebral body dislocation was detected in 1 case. The gap through posterior element and unfused facet joint in 6 lesions revealed the fracture line more accurately in comparison to radiographs.

MRI. Hypointense signal intensity of the vertebral lesion margins at the level of AL was noted in T1- and T2-weighted images. A decreased spinal canal was caused by anterior extradural tissue or posterior hypertrophic ligamentum flavum in 9 pseudarthrotic lesions. Among these lesions, obvious compression of the spinal cord was documented in 4 cases. Moreover, concurrent edematous paraspinal and soft tissue invasions were identified in the posterior element, which showed mixed or decreased intensity in $\mathrm{T} 2$-weighted images.

Mechanical trauma. Normal disc tissues were replaced by fibrous tissue without recognizable preserved nucleus pulposus. Proliferating fibroblastic tissue that extended into the disc-bone border or subchondral structure was observed in 11 cases. The hyaline cartilage was replaced by fibrous

Personal non-commercial use only. The Journal of Rheumatology Copyright @ 2019. All rights reserved. 
Table 1. General information and clinical data $(\mathrm{n}=17)$.

\begin{tabular}{|c|c|c|c|c|c|c|c|c|}
\hline Patient & Age/Sex & Level of AL & GK & Kyphosis* & $\mathrm{NF}$ & Duration, yrs & Location & Surgery \\
\hline 1 & $60 / \mathrm{F}$ & $\mathrm{T} 10 / 11, \mathrm{~T} 11 / 12$ & $33^{\circ}$ & - & Intact & $30+$ & Above & ADI \\
\hline 2 & $29 / \mathrm{M}$ & $\mathrm{T} 12 / \mathrm{L} 1$ & $45^{\circ}$ & - & Frankel D & $10+$ & Apex & $\mathrm{PTP}+\mathrm{AF}$ \\
\hline 3 & $52 / \mathrm{M}$ & T12/L1 & $80^{\circ}$ & + & Intact & $10+$ & Apex & $\mathrm{PTP}+\mathrm{AF}$ \\
\hline 4 & $37 / \mathrm{F}$ & T12/L1 & $106^{\circ}$ & + & Intact & 5 & Apex & $\mathrm{PTP}+\mathrm{AF}$ \\
\hline 5 & $32 / \mathrm{M}$ & $\mathrm{T} 11 / 12$ & $65^{\circ}$ & + & Intact & $5+$ & Apex & $\mathrm{PTP}+\mathrm{AF}$ \\
\hline 6 & $41 / \mathrm{M}$ & $\mathrm{T} 11 / 12$ & $95^{\circ}$ & + & Frankel D & $10+$ & Apex & $\mathrm{PTP}+\mathrm{AF}$ \\
\hline 7 & $40 / \mathrm{M}$ & $\mathrm{T} 11 / 12$ & $78^{\circ}$ & + & Intact & $10+$ & Apex & $\mathrm{PTP}+\mathrm{AF}$ \\
\hline 8 & $57 / \mathrm{M}$ & T12/L1 & $63^{\circ}$ & + & Intact & 20 & Apex & $\mathrm{PTP}+\mathrm{AF}$ \\
\hline 9 & $48 / \mathrm{M}$ & T9/10 & $57^{\circ}$ & + & Intact & 2 & Below & ADI \\
\hline 10 & 46/M & T12/L1 & $73^{\circ}$ & + & Intact & $10+$ & Above & PTP \\
\hline 11 & $43 / \mathrm{M}$ & $\mathrm{L} 2 / 3$ & $66^{\circ}$ & + & Intact & $10+$ & Below & PTP \\
\hline 12 & $35 / \mathrm{M}$ & $\mathrm{T} 10 / 11$ & $48^{\circ}$ & - & Intact & $10+$ & Apex & ADI \\
\hline 13 & $43 / \mathrm{F}$ & $\mathrm{L} 3 / 4$ & $42^{\circ}$ & - & Intact & 17 & Apex & PTP \\
\hline 14 & $54 / \mathrm{M}$ & $\mathrm{L} 4 / 5$ & $60^{\circ}$ & + & Frankel D & 15 & Below & PTP \\
\hline 15 & $54 / \mathrm{M}$ & $\mathrm{T} 12 / \mathrm{L} 1$ & $110^{\circ}$ & + & Intact & 10 & Apex & PI \\
\hline 16 & $46 / \mathrm{M}$ & $\mathrm{T} 11 / 12$ & $45^{\circ}$ & - & Intact & 2 & Apex & PIF \\
\hline 17 & $34 / \mathrm{M}$ & T12/L1 & $114^{\circ}$ & + & Intact & 11 & Apex & PI \\
\hline
\end{tabular}

* Kyphosis: GK > 50. AL: Andersson lesion; GK: global kyphosis; NF: neurological function; ADI: anterior debridement and instrumentation; Frankel D: Frankel classification grade D (fair to good motor function below injury level); PTP: pedicle subtraction osteotomy through pseudarthrosis; AF: supplemental anterior fusion; PI: pedicle subtraction osteotomy and instrumentation; PIF: posterior instrumentation and fusion; Apex: apical region of GK; Above: above the apex of GK; Below: below the apex of GK.

materials, showed degenerative changes and developed into fibrocartilage in 6 lesions (Figure 1A). In the inner portion of the fibrous tissue and fibrocartilage, extensive fibrinoid necrosis was frequently observed and associated with a loss of chondrocyte nuclei (Figure 2B). Occasionally, necrotic bone fragments enclosed within collagenous tissue in the peripheral disc space indicated a previous traumatic process. In addition, hemosiderosis was detected in the outermost part

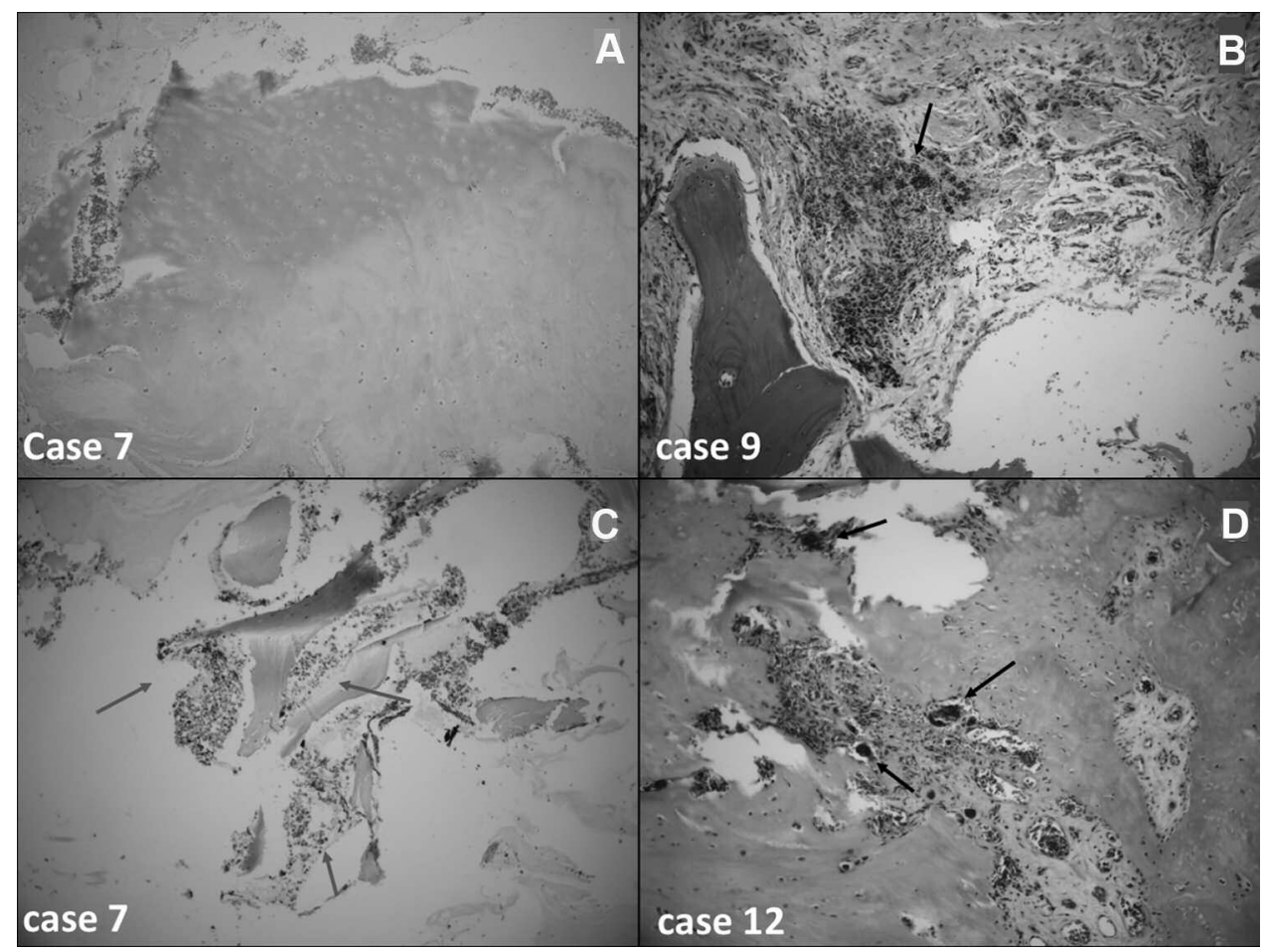

Figure 1. Evidence of mechanical trauma. A. Hyaline cartilage replaced by fibrocartilage. B. Hemosiderin deposit (arrow). C. Scanty perivascular inflammatory cellular infiltration (arrows). D. Active osteoclastic resorption and widespread osteoblasts infiltration (arrows) at the disc-bone border. (A-C H\&E; original magnification $\times 10$. D. $\times 20)$.

Personal non-commercial use only. The Journal of Rheumatology Copyright (C) 2019. All rights reserved. 


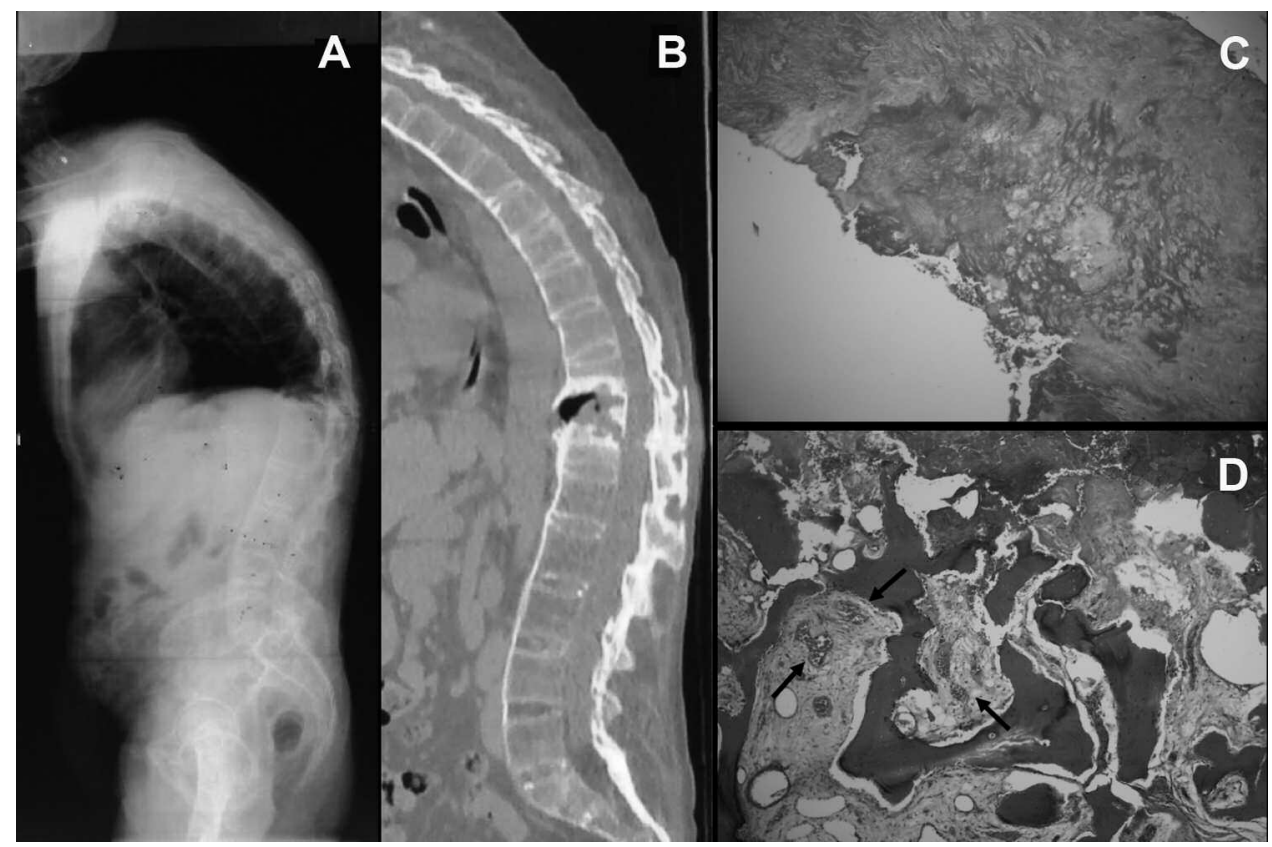

Figure 2. A 41-year-old male with pseudarthrosis at the apex (T10/11). Completely ossified anterior longitudinal ligaments (A) above and (B) below pseudarthrosis. C. Extensive fibrinoid necrosis implied that excessive stress had been applied to the skipped segment. D. Fibrous tissue bearing capillary congeries indicated an ongoing reparative process (arrows). (C-D H\&E, $\times 10)$.

of the fibrocartilage in 4 lesions, demonstrating old and repetitive hemorrhage caused by a previous trauma (Figure 1B).

Fibrous repair. The observed disc changes were consistent across all the lesions. In the intervertebral disc space, the normal structure was completely converted into hypovascular edematous fibrous scar tissue. In 7 lesions, proliferating reparative fibrous tissue had grown from the bone marrow, leading to the erosion and breach of the endplate. Moreover, the increased reversal lines disrupted the disc-bone border, making it unidentifiable. In an attempt to repair the posttraumatic necrotic zones and degenerative cartilaginous tissue, multiple laminae-bearing vessels merged with collagen fibers extending into the vertebral body; these could be observed microscopically in 6 cases. The presence of capillary congeries also revealed that a typical fibrous repair process was ongoing (Figure 2D). Scanty and mild perivascular inflammatory cellular infiltration (e.g., by monocytes, lymphocytes, and plasma cells) was noted in only 2 cases (Figure 1C).

Bony repair. Osteogenesis was more likely to occur near the endplate region instead of the disc space. Focal active osteoclastic resorption is the initiating process of bony repair and was observed in nearly all cases (Figure 1D). Further, chondroid and osseous metaplasia, indicated by widespread infiltration by osteoblasts at the disc-bone border, were also helpful clues to the pathogenesis of pseudarthrosis. Capillary vessels were frequently noted in regions of active osteogenesis. In 5 lesions, clear calcium deposits were observed in the margins of regenerative fibrocartilage, implying that bone formation was an ongoing process. Woven bone was frequently encountered in these lesions, which was the evidence of fracture (Figure 3D).

\section{DISCUSSION}

Spinal pseudarthrosis is an uncommon but well-recognized condition that occurs in the late stage of $\mathrm{AS}^{8}$. The prevalence of $\mathrm{AL}$ ranges from 1 to $28 \% 11,12,13$. Since Andersson published the first report of AL in $1937^{2}$, various terms have been used to describe these lesions. An infectious origin was initially suggested by some authors because of the radiological resemblance between $\mathrm{AL}$ and spondylodiscitis ${ }^{14}$. However, this assumption was not widely accepted because positive cultures were not detected. In a study of 2 patients, Wholey, et $a l^{5}$ was the first to report relevant findings from biopsies. These data showed that the etiology of AL may involve a chronic inflammatory process (Table 2). In 1967, Hackenbroch ${ }^{15}$ also noted nonspecific inflammation with cell infiltration, which supported a primary inflammatory origin for AL. However, further histological investigations favoring an inflammatory etiology are rare. In 1972, Cawley, et al $^{11}$ categorized AL into 2 types, localized and extensive lesions. In previous studies, localized lesions were regarded as originating from inflammation and part of a normal AS course ${ }^{16}$. However, whether inflammatory or traumatic factors are important to the development of extensive lesions (so-called pseudarthrosis) remained unclear. Thus, in our study, we sought to correlate pathological and radiographic findings with inflammatory or traumatic etiologies to determine the

Personal non-commercial use only. The Journal of Rheumatology Copyright $\odot$ (2019. All rights reserved 


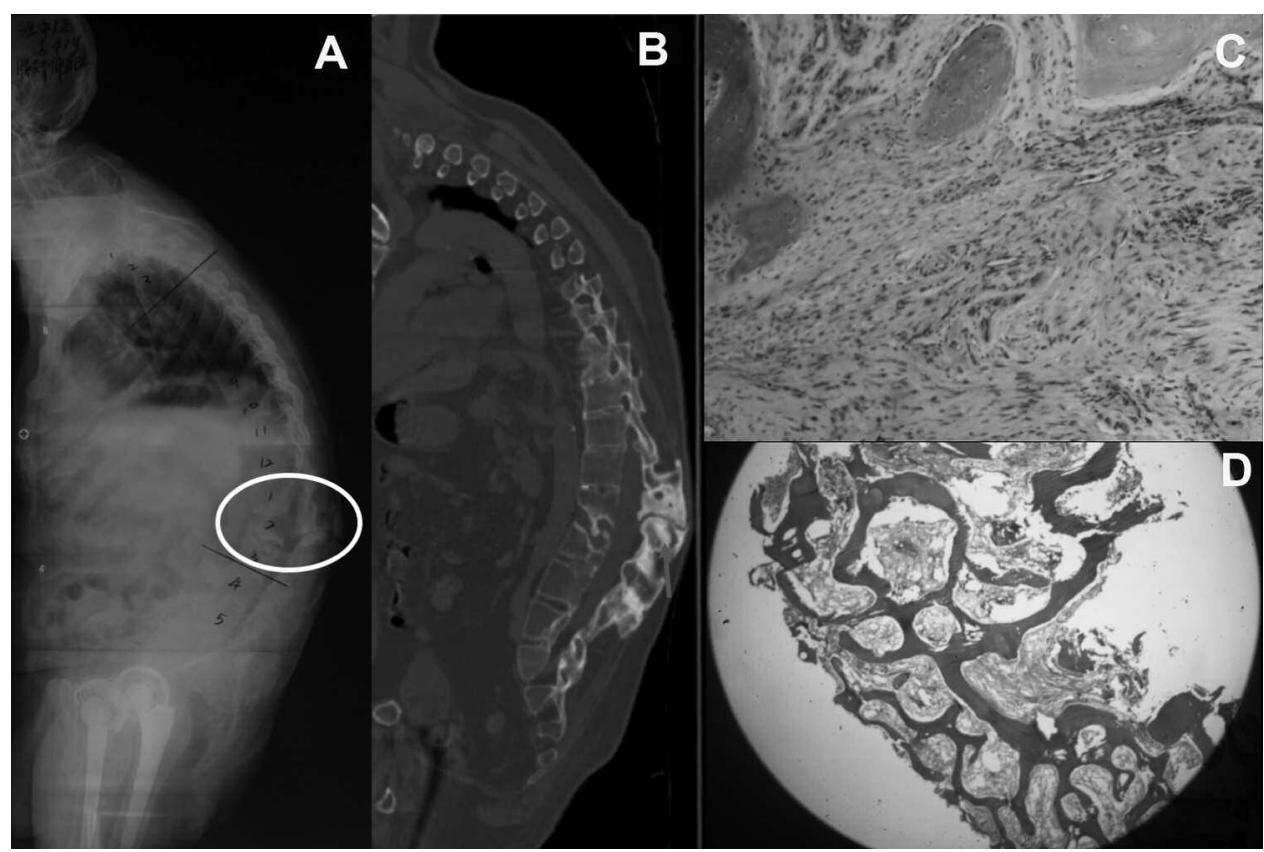

Figure 3. A 43-year-old female developed pseudarthrosis (A) below the apical region (L2/3). B. An acute fracture through the posterior elements was noted at the ankylosed spinal column. C. Fibrous tissue growing from the bone marrow indicated an active bony reparative process. D. Detected hemosiderin deposition in the outermost part of the fibrocartilage exhibited evidence of old hemorrhage. Woven bone revealed ongoing osteogenesis. (C-D H\&E, $\times 10)$.

Table 2. Overview of histological appearances of tissue obtained from AL reported over the past 60 years.

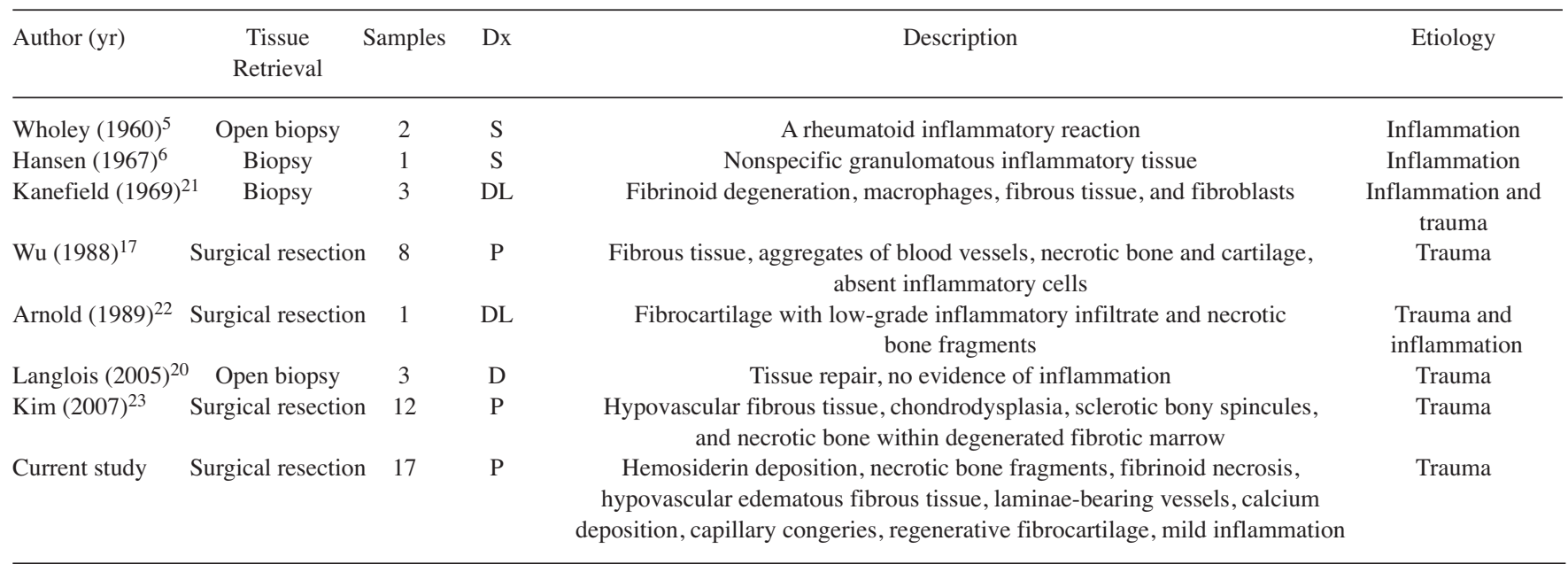

Dx: diagnosis; S: spondylodiscitis; DL: destructive lesion; D: discitis; P: pseudarthrosis; AL: Andersson lesions.

specific pathogenesis of pseudarthrosis (Supplementary Tables 2 and 3, available with the online version of this article).

In our current study, a pseudarthrosis located at the apical region of GK was noted in 12 out of 17 patients. It is conceivable that in these patients, the apical region of their fixed and kyphotic spine was subjected to the highest level of stress ${ }^{16}$. As thoracolumbar kyphosis progressed in advanced AS, repeated stress may have led to the extensive and severe destruction of the discs and resulted in fatigue fractures ${ }^{8,17}$. Therefore, in fibrous tissue with mild inflammation, the inflammation was most active in the intervertebral disc space, where the normal structure of the disc was totally destroyed and unrecognizable in all of the lesions. These pathological observations are also strongly reflective of the reparative process that occurs after mechanical

$$
\text { Personal non-commercial use only. The Journal of Rheumatology Copyright @ } 2019 \text {. All rights reserved. }
$$


trauma ${ }^{16}$. Moreover, widespread cartilaginous degeneration and necrosis were identified in the lesions in these cases. These findings suggest that the tissues had been exposed to repetitive stress and that the fibrocartilage showed higher resistance than hyaline cartilage to trauma. In 7 cases, the spinal segments above and below the pseudarthrosis were completely ossified and showed bridging syndesmophytes anterior to the lesion. The movement of the ankylosed spine was focused on the isolated segment, consistent with "the last mobile segment principle" proposed by $\mathrm{Wu}$, et $a l^{17}$. The skipped segment was consequently exposed to abnormal and excessive stress because of these long lever arms, and this caused a mechanical failure similar to that observed in a long bone fracture. Fibrinoid necrosis was also observed in these cases under microscopic examination, revealing undue stress on the tissues in this area. Generally, the bone and disc space are more susceptible to shearing forces than compressive forces ${ }^{8}$. Persistent motion at the isolated segment and the loss of structural support caused by the destruction of the fibrous annulus and nucleus pulposus may continue to produce damage, and may hinder repair of the fracture, resulting in the development of an unstable pseudarthrosis. Sclerotic subchondral bone at the level of the pseudarthrosis is evidence of an unfused spinal segment ${ }^{17,18}$.

Apart from mechanical trauma at non-ankylosed segments, other mechanisms that could potentially lead to the formation of pseudarthrosis included an acute fracture through the ankylosed spine. Because the entire spine is osteoporotic and stiff in AS, a minor trauma can cause a transvertebral, discovertebral, or transdiscal fracture ${ }^{16}$. In agreement with previous studies, we found that a fracture might initially occur in the posterior column prior to the development of anterior discovertebral lesions, especially when the patient is in a hyperflexion position, because this causes the greatest amount of posterior tension force ${ }^{1,7,8}$. In our present study, a microfracture through a posterior element that exhibited unfused facet joints was identified in 6 patients. Hemosiderin deposits, scattered necrotic bone fragments, and active osteoclasts and osteoblasts were concurrently detected in these cases, which was consistent with an old fracture and the natural healing process ${ }^{16,17,19,20}$. In AS patients with a longstanding disease course, inflammation frequently weakens the bony structure, leading to the vertebral body being susceptible to mechanical trauma. Therefore, undue or excessive stress is more likely to develop a pseudarthrotic lesion. In this case, inflammation appears to be the cause of the formation of pseudoarthrosis; however, trauma should instead be regarded as the predominant factor. Further, scant and mild inflammation was frequently accompanied by capillary congeries or vessels and was regarded as a reaction to trauma that played a secondary role in the pathogenesis of pseudarthrosis in AS. These findings support the observations made by Fang, et $a l^{8}$. Hence, for patients with AS in the advanced or late stage, trauma, farm work, and heavy labor that might lead to the development of trauma-originated pseudarthrosis should be avoided.

Although the findings of our study would appear to be partially consistent with the previous studies, we acknowledge the strengths of our study. We were able to (1) analyze the detailed correlation between distinctive radiological features and pathological findings, making multiple mechanisms completely understandable; (2) assess more histological changes as indicators in comparison to the previous literatures, such as woven bone, regenerative fibrocartilage replacing hyaline cartilage, and the like, which can provide a comprehensive understanding of the exact etiology; (3) include the largest amount of tissue specimens and pathological sections, making the pathological observations more persuasive; and (4) exhibit natural history of pseudarthrosis by characteristic radiological data, showing a clear trauma-related process in the formation of pseudarthrosis (Figure 4). The limitations of our study were its retrospective design and a failure to identify an association between clinical data and pathological analysis. Also, the target group mainly involving apex lesions is indeed one of the limitations of our study. The lesions near the apex are also subject to undue stress apart from the apical region, and are likely to result in formation of pseudarthrosis. In our current study, it could be concluded that the formation of apex lesions in AS is attributed to traumatic etiology. However, there were only 5 cases with pseudarthrosis located outside the apical region and because of the small sample size, it was hard to draw a persuasive conclusion for these lesions. Therefore, a better analysis involving more cases with lesions around the apex should be conducted in future investigations to distinguish whether inflammatory or traumatic factors play an important role. Further study using molecular biological methods to investigate the etiology of this condition is certainly warranted.

The traumatic etiology of spinal pseudarthrosis in AS can be histologically confirmed by the presence of mechanical trauma, fibrous, and bony repair. Moreover, a traumatic origin can be established based on evidence of multiple biochemical mechanisms, such as those involved in the "last mobile segment principle," repetitive stress, and acute fracture. Finally, no pathological evidence of primary inflammatory origin was noted in these cases. Hence, pseudarthrosis is more likely to have originated as a result of mechanical trauma than inflammation.

\section{ONLINE SUPPLEMENT}

Supplementary material accompanies the online version of this article.

\section{REFERENCES}

1. Qian BP, Qiu Y, Wang B, Sun X, Zhu ZZ, Jiang J, et al. Pedicle subtraction osteotomy through pseudarthrosis to correct thoracolumbar kyphotic deformity in advanced ankylosing spondylitis. Eur Spine J 2012;21:711-8.

2. Bron JL, de Vries MK, Snieders MN, van der Horst-Bruinsma IE,

Personal non-commercial use only. The Journal of Rheumatology Copyright $\odot$ 2019. All rights reserved. 


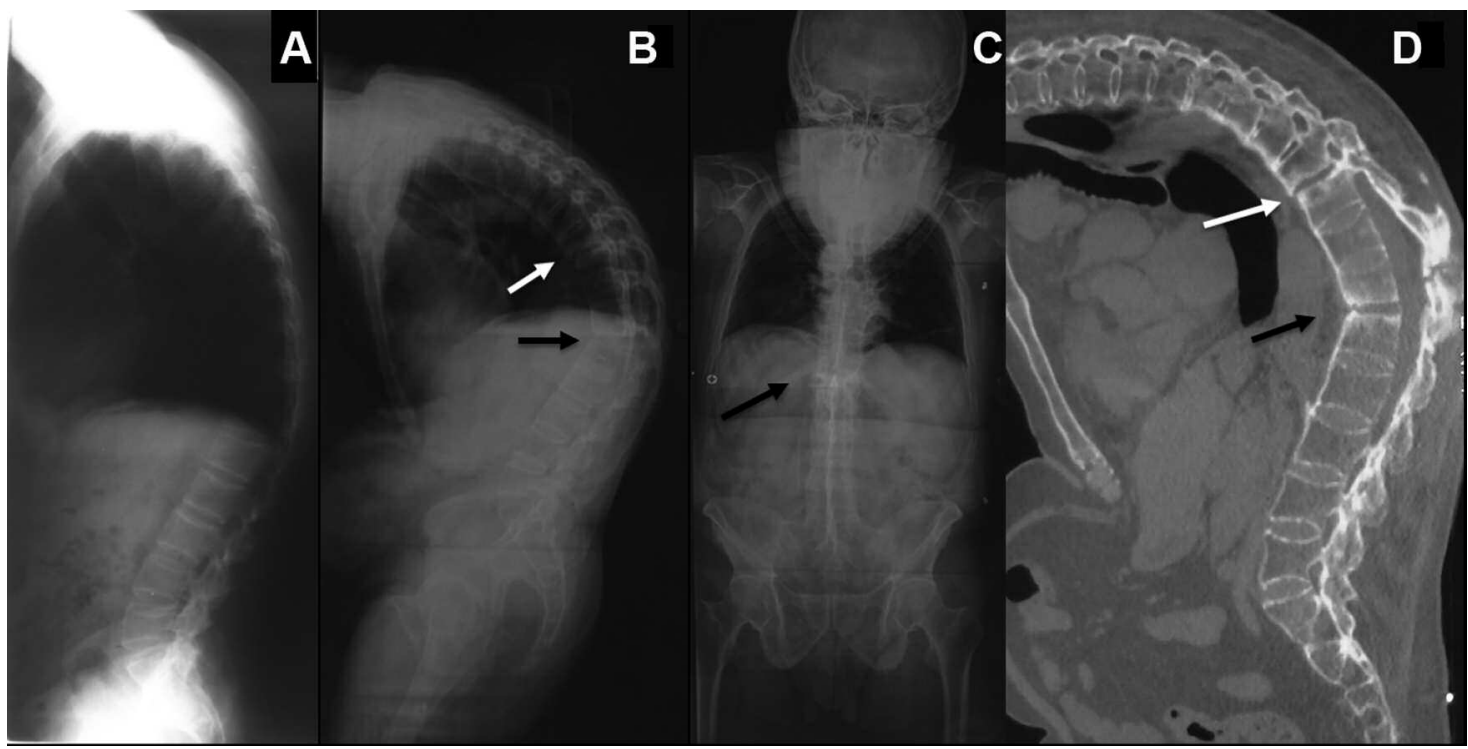

Figure 4. Natural history of pseudarthrosis. A. Initial visit of a 34-year-old male without detected pseudarthrosis. At 11-year followup, the patient had developed pseudarthrosis [B-D, T9/10 (white arrows) and T12/L1 (black arrows)] and exhibited progression of thoracolumbar kyphosis. D. CT sagittal reconstruction image showed the formation of pseudarthrosis involving 3 columns (black arrow). CT: computed tomography.

van Royen BJ. Discovertebral (Andersson) lesions of the spine in ankylosing spondylitis revisited. Clin Rheumatol 2009;28:883-92.

3. Kabasakal Y, Garrett SL, Calin A. The epidemiology of spondylodiscitis in ankylosing spondylitis - a controlled study. Br J Rheumatol 1996;35:660-3.

4. Scarpa R. Discovertebral erosions and destruction in psoriatic arthritis. J Rheumatol 2000;27:975-8.

5. Wholey MH, Pugh DG, Bickel WH. Localized destructive lesions in rheumatoid spondylitis. Radiology 1960;74:54-6.

6. Hansen ST Jr, Taylor TK, Honet JC, Lewis FR. Fracture-dislocations of the ankylosed thoracic spine in rheumatoid spondylitis.

Ankylosing spondylitis, Marie-Strümpell disease. J Trauma 1967;7:827-37.

7. Coste F, Delbarre F, Cayla J, Massias P, Beaslay E. [Destructive spondylitis in ankylosing spondylarthritis.] [Article in French] Presse Med 1963;71:1013-6.

8. Fang D, Leong JC, Ho EK, Chan FL, Chow SP. Spinal pseudarthrosis in ankylosing spondylitis. Clinicopathological correlation and the results of anterior spinal fusion. J Bone Joint Surg Br 1988;70:443-7.

9. Molnar C, Scherer A, Baraliakos X, de Hooge M, Micheroli R, Exer $\mathrm{P}$, et al; Rheumatologists of the Swiss Clinical Quality Management Program. TNF blockers inhibit spinal radiographic progression in ankylosing spondylitis by reducing disease activity: results from the Swiss Clinical Quality Management cohort. Ann Rheum Dis 2018;77:63-9.

10. Haroon N, Kim TH, Inman RD. NSAIDs and radiographic progression in ankylosing spondylitis: Bagging big game with small arms? Ann Rheum Dis 2012;71:1593-5.

11. Cawley MI, Chalmers TM, Kellgren JH, Ball J. Destructive lesions of vertebral bodies in ankylosing spondylitis. Ann Rheum Dis 1972;31:345-58.

12. Rasker JJ, Prevo RL, Lanting PJ. Spondylodiscitis in ankylosing spondylitis, inflammation or trauma? A description of six cases. Scand J Rheumatol 1996;25:52-7.
13. Park WM, Spencer DG, McCall IW, Ward J, Buchanan WW, Stephens WH. The detection of spinal pseudarthrosis in ankylosing spondylitis. Br J Radiol 1981;54:467-72.

14. Eschelman DJ, Beers GJ, Naimark A, Yablon I. Pseudoarthrosis in ankylosing spondylitis mimicking infectious diskitis: MR appearance. AJNR Am J Neuroradiol 1991;12:1113-4

15. Hackenbroch MH. [Localized osteolytic processes in spondylarthritis ankylopoietica.] [Article in German] Z Orthop Ihre Grenzgeb 1967;103:23-33.

16. Dihlmann W, Delling G. Disco-vertebral destructive lesions (so-called Andersson lesions) associated with ankylosing spondylitis. Skeletal Radiol 1978;3:10-6.

17. Wu PC, Fang D, Ho EK, Leong JC. The pathogenesis of extensive discovertebral destruction in ankylosing spondylitis. Clin Orthop Relat Res 1988;230:154-61

18. Kurugoglu S, Mihmanli I, Kanberoglu K. Destructive diskovertebral lesions in ankylosing spondylitis: appearance on magnetic resonance imaging. South Med J 2001;94:837-41.

19. Park YS, Kim JH, Ryu JA, Kim TH. The Andersson lesion in ankylosing spondylitis: distinguishing between the inflammatory and traumatic subtypes. J Bone Joint Surg Br 2011;93:961-6.

20. Langlois S, Cedoz JP, Lohse A, Toussirot E, Wendling D. Aseptic discitis in patients with ankylosing spondylitis: a retrospective study of 14 cases. Joint Bone Spine 2005;72:248-53.

21. Kanefield DG, Mullins BP, Freehafer AA, Furey JG, Horenstein S, Chamberlin WB. Destructive lesions of the spine in rheumatoid ankylosing spondylitis. J Bone Joint Surg Am 1969;51:1369-75.

22. Arnold MH, Brooks PM, Ryan M, Francis H. A destructive discovertebral lesion: septic discitis, ankylosing spondylitis, or rheumatoid arthritis? Clin Rheumatol 1989;8:277-81.

23. Kim KT, Lee SH, Suk KS, Lee JH, Im YJ. Spinal pseudarthrosis in advanced ankylosing spondylitis with sagittal plane deformity. Spine 2007;32:1641-7. 